\title{
TEMPERATURE DISTRIBUTION IN SOLIDS OF VARIABLE THERMAL PROPERTIES HEATED BY MOVING HEAT SOURCES*
}

\author{
BY \\ li. J. (iROKH, I: A. TRABANT, AND G. A. HAWKINS- \\ Purdue Tiniversity
}

\begin{abstract}
The temperature distribution in rods, plates, and infinite solids heated by moving plance, line. and point heat sources is obtained for the quasi-stationary state. The thermal properties of the solid can vary as a linear, parabolic, or some other convenient function of temperature as long as the thermal diffusivity of the material remains nearly constant.
\end{abstract}

Introduction. The theory of moring heat sources has been applied in the main to weiding problems; however, in recent years the theory has been extended in applications to extrusion, flame hardening, gun barrels, and determinations of thermal diffusivities. The theory as usually presented assumes that thermal properties are independent of temperature, that heat generation due to phase change or Joule heating is negligible, and that the source moves in such a manner that the temperature field about the moving heat source is independent of time, the so-called quasi-stationary state [1]. ${ }^{1}$ It is the purpose of this paper to present a theory which accounts, at least in part, for the variation of thermal properties with temperature. The results obtained are applicable to certain one, two, and three dimensional problems where the heat source can conveniently be represented by a moving plane, line, or point.

Fundamental equations. The partial differential equation for heat conduction in a moving isotropic solid the thermal properties of which are functions of temperature and/or position and where any heat generation within the solid is negligible can be written as

$$
\nabla \cdot(k \nabla T)=\rho c \frac{D T}{D \tau}
$$

where $T$ is the temperature and a function of the coordinates $x, y, z$ in a fixed Cartesian coordinate system and time $\tau ; k$ is thermal conductivity and a function of temperature and/or coordinates; $\rho$ is density and a function of temperature and/or coordinates; $c$ is specific heat and a function of temperature and/or coordinates; and $D T / D \tau$ is the substantial, fluid, or total derivative of temperature. Assuming linear motion for the solid as shown in Fig. 1 and a quasi-stationary temperature field, this equation can be reduced to

$$
\nabla \cdot(k \nabla T)=-\rho c v \frac{\partial T}{\partial x},
$$

where $v$ is the speed of the solid with respect to the fixed Cartesian coordinate system. The source will be considered fixed as a plane, line, or point about the origin of the fixed coordinate system. It is of no consequence whether the source is considered fixed in space and the solid moves, or, the solid is considered fixed in space and the source moves.

*Received July 19, 1954.

Niumbers in brackets refer to the Bibliography at the end of the paper. 
In the latter case, the fundamental equation may be expressed as Eq. (1) through the use of a suitable transformation of variable [3].

Heat sources are assumed to be planes, lines, or points. A plane source is a plane surface of area $A$ which emits $q$. units of energy per unit time in its normal direction $n$ as

$$
q_{.}=-k A \frac{\partial T}{\partial n} \text {. }
$$

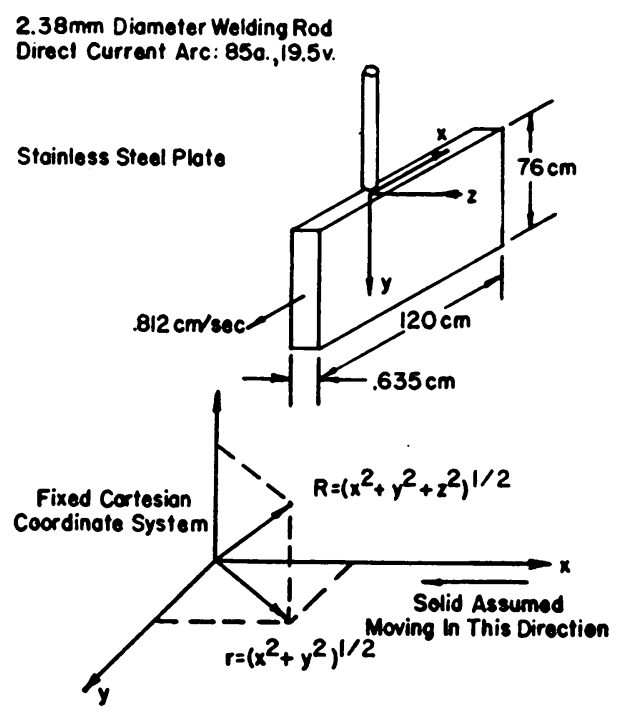

Fig. 1. Coordinate system.

A line source is a segment parallel to the $z$ axis of length $L$ which emits $q_{L}$ units of energy per unit time as

$$
q_{L}=\operatorname{Lim}_{r \rightarrow 0}-2 \pi L r k \frac{\partial T}{\partial r},
$$

where $r=\left(x^{2}+y^{2}\right)^{1 / 2}$. A point source is defined to emit $q_{p}$ units of energy per unit time as

$$
q_{p}=\operatorname{Lim}_{R \rightarrow 0}-4 \pi R^{2} k \frac{\partial T}{\partial R},
$$

where $R=\left(x^{2}+y^{2}+z^{2}\right)^{1 / 2}$.

Other boundary or surface conditions will be considered to be of the adiabatic or "no flux" type. That is

which follows from

$$
\frac{\partial T}{\partial \xi}=0 \quad \xi= \pm \infty, \pm b
$$

$$
k \frac{\partial T}{\partial \xi}=0 \quad \xi= \pm \infty, \pm b,
$$

since $k>0$. The symbol $\xi$ represents $x, y, z, r$, or $R$; $b$ is a constant. 
The thermal properties of a solid are generally functions of temperature. It is convenient to assume that for those properties appearing in Eqs. (1) through (7)

$$
k=k_{0} f^{\prime}(T),
$$

$$
\rho c=(\rho c)_{0} f^{\prime}(T),
$$

where $k_{0}$ and $(\rho c)_{0}$ are positive constants and $f^{\prime}(T)$ is the temperature derivative of some function of temperature $f(T)$. It is assumed that $f(T)$ and $f^{\prime}(T)$ are differentiable and $f^{\prime}(T)$ is greater than zero to avoid violation of the Second Law of Thermodynamics. The particular convenience of these forms appeals, in any case, to experimental data. The efficacy of this form of assumption is shown in Fig. 2 for a stainless steel.
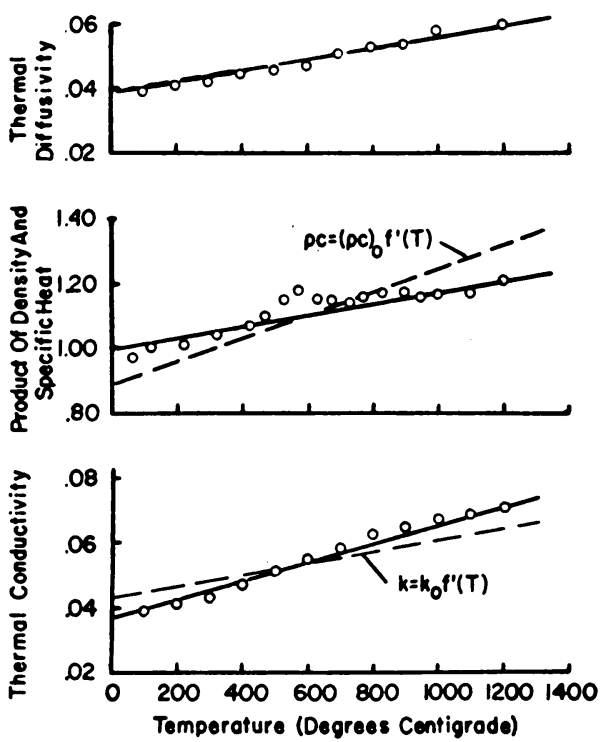

Fig. 2. Thermal conductivity, product of density and specific heat, and thermal diffusivity. $\circ$ Data of Powell [2], CGS units.

Generally,

$$
\frac{\partial f(T)}{\partial \xi}=f^{\prime}(T) \frac{\partial T}{\partial \xi} .
$$

This equality is used to simplify Eqs. (2) through (7); they become respectively

$$
\begin{aligned}
\nabla^{2} f(T)=-2 \lambda v \frac{\partial f(T)}{\partial x}, & \quad(11) \quad q_{0}={ }_{-}-k_{0} A \frac{\partial f(T)}{\partial n}, \\
q_{L} & =\operatorname{Lim}_{r \rightarrow 0}-2 \pi r k_{0} L \frac{\partial f(T)}{\partial r}, \\
q_{P} & =\operatorname{Lim}_{R \rightarrow 0}-4 \pi R^{2} k_{0} \frac{\partial f(T)}{\partial R}, \\
\frac{\partial f(T)}{\partial \xi} & =0, \quad \xi= \pm \infty, \pm b,
\end{aligned}
$$

where $\lambda=\frac{1}{2} \alpha$ and $\alpha$ is the thermal diffusivity $k / \rho c$. 
The reader is referred to papers by Rosenthal [3] and Rosenthal and Cameron [4] for solutions of the following boundary value problems after the above transformations have been made.

One dimensional heat flow problem. If it is assumed that heat flows in the $x$ direction only, then

$$
\frac{\partial}{\partial x}\left\{k_{0} f^{\prime}(T) \frac{\partial T}{\partial x}\right\}=-(\rho c)_{0} v f^{\prime}(T) \frac{\partial T}{\partial x} .
$$

Let the boundary conditions be

$$
\begin{array}{rl}
k_{0} f^{\prime}(T) \frac{\partial T}{\partial x}=0 & x= \pm \infty, \\
-k_{0} A \frac{\partial T}{\partial x}=q . & x=0,
\end{array}
$$

and let the initial temperature of the solid be $T_{i}$ :

$$
T=T_{i} \quad x=+\infty .
$$

From the foregoing transformations, this problem reduces to

$$
\begin{gathered}
\frac{\partial^{2} f(T)}{\partial x^{2}}=-2 \lambda v \frac{\partial f(T)}{\partial x}, \\
\frac{\partial f(T)}{\partial x}=0 \quad x= \pm \infty, \\
-k_{0} A \frac{\partial f(T)}{\partial x}=q . \quad x=0, \\
f(T)=f\left(T_{i}\right) \quad x=+\infty .
\end{gathered}
$$

The solution to this problem is

$$
\begin{array}{ll}
f(T)-f\left(T_{i}\right)=\frac{q_{i}}{A(\rho c)_{0} v} \cdot \exp (-2 \lambda v x) & x \geq 0, \\
f(T)-f\left(T_{i}\right)=\frac{q_{i}}{A(\rho c)_{0} v} & x \leq 0 .
\end{array}
$$

If $f^{\prime}(T)=1+m T$, where $m$ is a positive constant; that is, the thermal conductivity and product of density and specific heat are linear functions of temperature; then Eqs. (21) and (22) reduce to

$$
\begin{array}{ll}
T=\frac{1}{m}\left\{\left[\frac{2 q_{\mathrm{v}} m}{A(\rho c)_{0} v} \exp (-2 \lambda v x)+\left(1+m T_{i}\right)^{2}\right]^{1 / 2}-1\right\} & x \geq 0, \\
T=\frac{1}{m}\left\{\left[\frac{2 q_{\mathrm{s}} m}{A(\rho c)_{0} v}+\left(1+m T_{i}^{2}\right)\right]^{1 / 2}-1\right\} & x \leq 0 .
\end{array}
$$

The solution corresponds to the temperature distribution in an infinite rod or solid due to a moving plane source.

Two dimensional heat flow problems. (a) The infinite plate. If heat flows in the $x$ and $y$ directions from a line source along the $z$ axis, then

$$
\frac{\partial^{2} f(T)}{\partial x^{2}}+\frac{\partial^{2} f(T)}{\partial y^{2}}=-2 \lambda v \frac{\partial f(T)}{\partial x} .
$$


Further, let

$$
\begin{gathered}
\frac{\partial f(T)}{\partial r}=0 \quad r=\infty, \\
q_{L}=\operatorname{Lim}_{r \rightarrow 0}-2 \pi r L k_{0} \frac{\partial f(T)}{\partial x},
\end{gathered}
$$

and the initial temperature of the plate be $T_{i}$ so that

$$
f(T)=f\left(T_{\mathrm{i}}\right) \quad r=\infty .
$$

The solution to this problem is

$$
f(T)-f\left(T_{i}\right)=\frac{q_{L}}{2 \pi k_{0} L} \exp (-\lambda v x) K_{0}(\lambda v r),
$$

where $K_{0}(\lambda v r)$ is a modified Bessel function of the second kind, zero order, with argument $\lambda v r$. If the thermal properties are certain linear functions of temperature; that is, $f^{\prime}(T)=1+m T$; then

$$
T=\frac{1}{m}\left\{\left[\frac{q_{L} m}{\pi L k_{0}} \exp (-\lambda v x) K_{0}(\lambda v r)+\left(1+m T_{i}\right)^{2}\right]^{1 / 2}-1\right\} .
$$

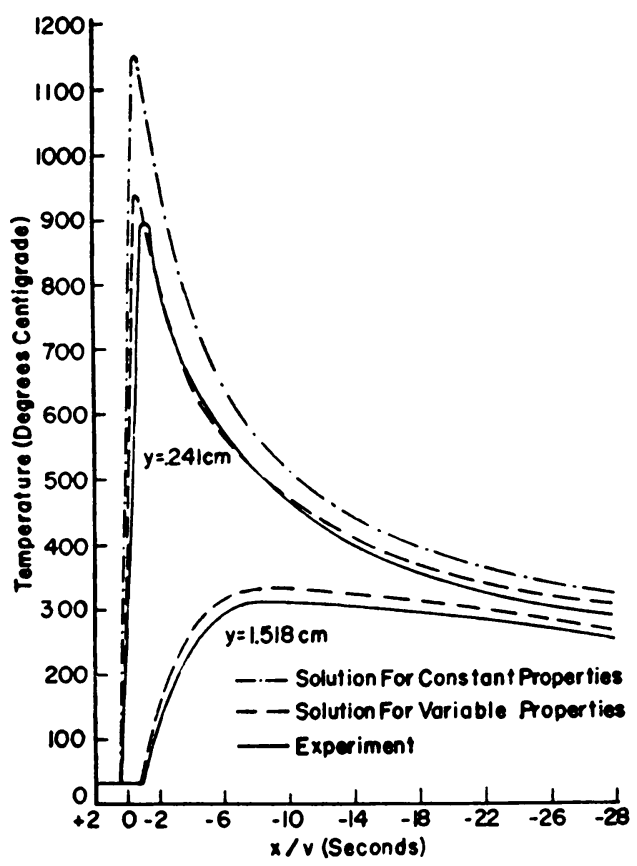

Fig. 3. Application of theory Eq. 30 to experiment.

This equation is of interest in regard to welding thin, stainless steel plates. An application of this equation to welding can be found elsewhere [5]. A synopsis of this is shown in Figs. 1 and 3. 
(b) The finite plate. In contradistinction to the previous case, if the plate is of width $b$ in the $y$ direction then

$$
\begin{array}{ll}
\frac{\partial f(T)}{\partial y}=0 & y=0, b, \\
\frac{\partial f(T)}{\partial x}=0 & x= \pm \infty .
\end{array}
$$

The solution to this case, obtained by Rosenthal using the method of images, is

$$
f(T)-f\left(T_{i}\right)=\frac{q_{L}}{\pi L k_{0}} \exp (-\lambda v x) \sum_{-\infty}^{+\infty} K_{0}\left(\lambda v r_{n}\right),
$$

where $r_{n}=\left(x+[y \pm 2 n b]^{2}\right)^{1 / 2}$. Again, considering the linear type variation of thermal properties,

$$
T=\frac{1}{m}\left\{\left[\frac{2 q_{L} m}{\pi L k_{0}} \exp (-\lambda v x) \sum_{-\infty}^{+\infty} K_{0}(\lambda v r)+\left(1+m T_{i}\right)^{2}\right]^{1 / 2}-1\right\} .
$$

Three dimensional heat flow problems. (a) The infinite solid. If heat flows in the $x, y$, and $z$ directions from a point source moving along the $x$ axis, then

$$
\nabla^{2} f(T)=-2 \lambda v \frac{\partial f(T)}{\partial x}
$$

The boundary conditions will be

$$
\begin{gathered}
\frac{\partial f(T)}{\partial R}=0 \quad R=\infty \\
q_{P}=\operatorname{Lim}_{R \rightarrow 0}-4 \pi R^{2} k_{0} \frac{\partial f(T)}{\partial R}
\end{gathered}
$$

and the initial temperature of the solid will be $T_{i}$ or

$$
f(T)=f\left(T_{i}\right) \quad R=\infty .
$$

The solution to this problem is

$$
f(T)-f\left(T_{i}\right)=\frac{q_{P}}{4 \pi k_{0} R} \exp (-\lambda v x) \exp (-\lambda v R) .
$$

If the thermal properties are certain linear functions of temperature, then

$$
T=\frac{1}{m}\left\{\left[\frac{q_{P} m}{2 \pi k_{0} R} \exp (-\lambda v x) \exp (-\lambda v R)+\left(1+m T_{i}\right)^{2}\right]^{1 / 2}-1\right\} .
$$

(b) The finite solid. If the solid is of infinite extent in the $x$ and $y$ directions, but bounded by the planes $z=0$ and $z=g$; that is, the boundary conditions are

$$
\begin{gathered}
\frac{\partial f(T)}{\partial r}=0 \quad r=\infty, \\
\frac{\partial f(T)}{\partial z}=0 \quad z=0, g, \\
f(T)=f\left(T_{i}\right) \quad r=\infty,
\end{gathered}
$$


then it can be shown that the solution to this problem is

$$
f(T)-f\left(T_{i}\right)=\frac{q_{P}}{2 \pi k_{0}} \exp (-\lambda v x) \sum_{-\infty}^{+\infty} \frac{\exp \left(-\lambda v R_{n}\right)}{R_{n}},
$$

where $R_{n}=\left[x^{2}+y^{2}+(z \pm 2 n g)^{2}\right]^{1 / 2}$.

(c) The solid bounded by a cylinder. If the solid is of infinite length in the $x$ direction and is bounded in the $y$ and $z$ directions by a cylinder of radius $a$ concentric with the $x$ axis, another solution is available in rather complicated form. The cylindrical surface is assumed insulated and a point source moves along the $x$ axis.

Then

$$
\begin{gathered}
\nabla^{2} f(T)=-2 \lambda v \frac{\partial f(T)}{\partial x}, \\
\frac{\partial f(T)}{\partial \xi}=0 \quad \xi=\left(y^{2}+z^{2}\right)^{1 / 2}=a, \\
\frac{\partial f(T)}{\partial x}=0 \quad x= \pm \infty, \\
q_{D}=\operatorname{Lim}_{R \rightarrow 0}-4 \pi R^{2} k_{0} \frac{\partial f(T)}{\partial R}, \\
f(T)=f\left(T_{i}\right) \quad x= \pm \infty .
\end{gathered}
$$

The reader is referred to the original paper of Rosenthal and Cameron [4] for a discussion of the appropriate solution.

It is to be noted that, in all the above cases of one, two, and three dimensional conduction, only linear types of properties were considered. The method outlined is able to account for any variation of properties as long as Eqs. (8) and (9) are valid. However, the use of more accurate approximating functions requires solutions of high degree algebraic or trigonometric equations.

Summary. A method is outlined to account for certain variations of thermal properties of a solid when heated by a moving heat source. Variations considered are of the type where thermal conductivity and the product of density and specific heat are similar functions of temperature; or, where these properties vary but their ratio, the thermal diffusivity, is constant. The theory is applicable to one, two, and three dimensional cases of heat flow from a plane, line, and point moving sources.

It is felt that the theory furnishes a useful approximation to certain real systems. The theory has been verified in the particular case of welding thin stainless steel plates.

\section{BIBLIOGRAPHY}

1. M. Jakob, Heat transfer, John Wiley and Sons, 1949

2. R. W. Powell and co-workers, The physical properties of a series of steels, J. Iron and Steel Instit., 154, 83 (1946)

3. D. Rosenthal, The theory of moving sources of heat and its application to metal treatments, Trans. A.S.M.E. 68, 849 (1946)

4. D. Rosenthal and R. H. Cameron, Temperature distribution in cylinder heated by point source moving along its axis, Trans. A.S.M.E. 69, 961 (1947)

5. R. J. Grosh, Temperature distribution in welding, Ph.D. dissertation, Purdue University, August 1953 